\title{
ARTíCULO \\ Influencia de El Niño Oscilación del Sur en la disponibilidad y abundancia de recursos hidrobiológicos de la pesca artesanal en Ica, Perú
}

El Niño Southern Oscillation driven fluctuations in the availability and abundance of artisanal fishery resources in Ica, Peru

\section{Grant D. Adams ${ }^{1,2}$ y Daniel Flores ${ }^{1}$}

\begin{abstract}
'Laboratorio Costero de Pisco, Instituto del Mar del Perú IMARPE, Av. Los Libertadores A-12, Urb. El Golf, Paracas, Ica, Perú. grantadams60091@gmail.com

${ }^{2}$ Peace Corps Peru, 132 Vía Láctea, Surco, Perú

Abstract.- El Niño Southern Oscillation (ENSO) produces ecosystem-wide changes and plays a large role in the productivity of the Southeast Pacific ocean. Artisanal fisheries, an important source of income and food stability, are greatly affected by ENSO. The relationship between ENSO climate variation and the abundance of previously unstudied commercially important species to the artisanal fishery in Ica, Peru was analyzed using generalized additive models fitted to catch per unit effort (CPUE) data. We found that the immediate availability of Peruvian rock seabass (Paralabrax humeralis), Lorna drum (Sciaena deliciosa) and Minor stardrum (Stellifer minor) increased during cold water La Niña events. ENSO also produced long-term changes in the abundance of fisheries resources up to two years after ENSO events. In the case of Cabinza grunt (Isacia conceptionis), CPUE was elevated 6 months to two years after La Niña events. While for other species like Flathead grey mullet (Mugil cephalus) and P. humeralis, CPUE decreased between six months and two years after La Niña events. However, variation in the deviance explained between models indicates that the relative importance of ENSO as an environmental driver is species specific. This information can be used to assist in the development of management strategies of commercially important species in the Pacific Southeast and inform on the impacts of climate variation on artisanal fisheries. However, further study is warranted to examine the underlying mechanisms.
\end{abstract}

Key words: El Niño Southern Oscillation, artisanal fisheries, generalized additive models, climate variation, Peru

Resumen.- El Niño Oscilación del Sur (ENOS) produce grandes cambios en el ecosistema marino y juega un importante papel en la dinámica biológica de los recursos hidrobiológicos del Océano Pacífico Sudoriental. La pesquería artesanal es una fuente importante de ingresos económicos y seguridad alimentaria que se ve fuertemente afectada por el ciclo de ENOS. La relación entre ENOS y la disponibilidad y abundancia de especies de importancia en la pesquería artesanal en Ica, Perú fueron analizados usando modelos aditivos generalizados (GAMs) ajustados a datos de captura por unidad de esfuerzo (CPUE). Se determinó que la disponibilidad inmediata de cabrilla (Paralabrax humeralis), lorna (Sciaena deliciosa) y mojarrilla (Stellifer minor) fueron desfavorecidos por eventos fríos de La Niña. También ENOS cambió la abundancia de recursos pesqueros hasta dos años después del evento. En el caso de cabinza (Isacia conceptionis), su CPUE se incrementó desde los 6 meses hasta los dos años tras eventos de La Niña; mientras que para P. humeralis y lisa (M ugil cephalus), los eventos de La Niña desfavorecieron la presencia de mayores tasas de CPUE entre 6 meses y dos años después. Sin embargo, la variación de la desviación explicada entre los modelos indica que la importancia relativa de ENOS como un controlador ambiental dependiente de la especie. Esta información puede ser utilizada para desarrollar estrategias de manejo de especies de importancia comercial en el Pacífico Sudoriental e informar sobre los impactos de la variación climática sobre la pesca artesanal. No obstante, más información es necesaria para entender los mecanismos subyacentes.

Palabras clave: El Niño Oscilación del Sur, pesquería artesanal, modelos aditivos generalizados, variación climática, Perú

\section{INTRODUCCIÓN}

El Niño Oscilación Sur (ENOS) constituye una de las manifestaciones de variabilidad ambiental más conspicuas, afectando al ecosistema global, y con ello a los recursos marinos y sus pesquerías (Acha et al. 2004, Bakun \& Weeks 2008, Espino \& Yamashiro 2012). ENOS cambia las condiciones ambientales como la concentración de masas de agua, intensidad de afloramiento, y temperatura (Swartzman et al. 2008, Su et al. 2011). Los cambios ambientales impactan en los recursos pesqueros y a los pescadores en 2 periodos de tiempo distintos ya sea; 1) cambiando la distribución vertical y horizontal de recursos a corto plazo; y por tanto, modificandola disponibilidad de recursos pesqueros, a los que a partir de ahora nombraremos 
como 'disponibilidad'; y/o, 2) cambiando la reproducción, reclutamiento, y biomasa de una especie en el largo plazo (Pauly \& Tsukayama 1987, Ñiquen \& Bouchon 2004, Taylor et al. 2008), o abundancia. Por ejemplo, El Niño 1997-1998 produjo una gran abundancia de concha de abanico, Argopecten purpuratus, en Pisco, Perú, gracias a la mayor actividad reproductiva debido a las temperaturas elevadas (Wolff et al. 2007). Mientras que, un intenso esfuerzo pesquero combinado con una fase de El Niño especialmente fuerte en 1972, causó el colapso de anchoveta en Perú, teniendo grandes repercusiones a la economía (Pauly \& Tsukayama 1987, Swartzman et al. 2008, Cahuin et al. 2009).

Perú es tal vez el país más afectado por ENOS (Pauly \& Tsukayama 1987, Ballón et al. 2008, Bakun \& Weeks 2008, Quiñones et al. 2010) teniendo una pesquería artesanal grande, importante y dinámica (Alfaro-Shigueto et al. 2010, INEI \&
PRODUCE 2012). La pesca artesanal en el Perú, está caracterizada por embarcaciones con una capacidad máxima de bodega de $32,6 \mathrm{~m}^{3}$ y hasta $15 \mathrm{~m}$ de longitud y empleando predominantemente la mano de obra (Alfaro-Shigueto et al. 2010). La pesquería artesanal es una fuente económica y de seguridad alimentaria muy importante (Guisan et al. 2002, Béné et al. 2007, Andrew \& Evans 2009), constituyéndose como una de las actividades principales en países en vías de desarrollo (Salas et al. 2007). A pesar de ello, no se cuenta con información cuantitativa actualizada sobre los impactos de ENOS sobre los recursos hidrobiológicos aprovechados por la pesquería artesanal en el Perú. La escasa información cuantitativa, que falta por razones como la ausencia de evaluación y la diversidad de estas pesquerías, disminuye la habilidad de gestionar la pesca artesanal de manera sostenible (Andrew \& Evans 2009, Cardinale et al. 2014).

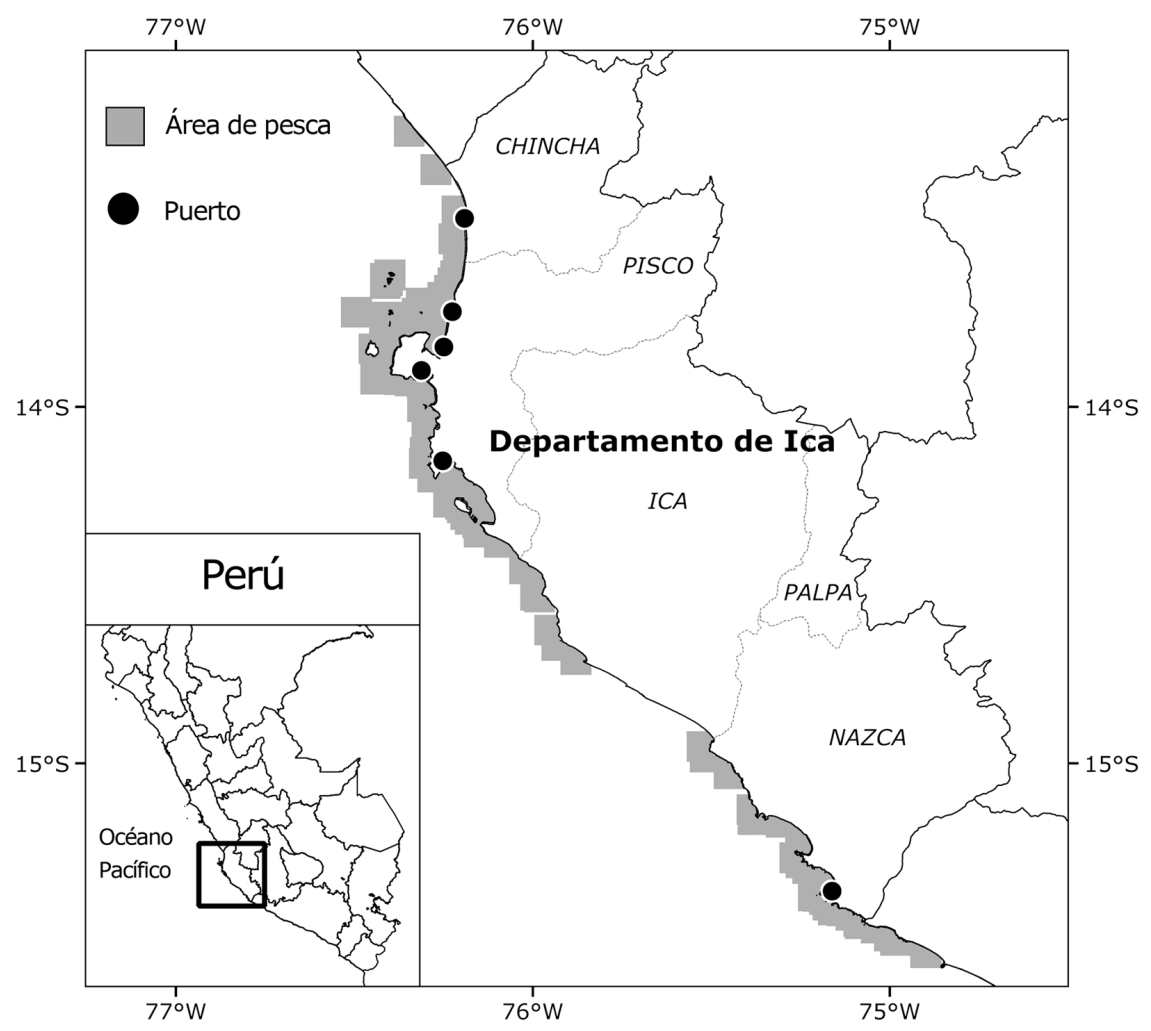

Figura 1. Mapa de las áreas de pesca de la flota artesanal en Ica, Perú, 2004-2013. Provincias en cursiva / Map of the fishing grounds of the artisanal fleet in Ica, Peru, 2004-2013. Provinces are noted in italics 
El departamento de Ica, Perú (Fig. 1), representa una zona de pesca artesanal importante con la segunda mayor población de pescadores del país (INEI \& PRODUCE2012), habiéndose reportado ya los efectos de ENOS sobre sus recursos y pesquerías (Wolff et al. 2007, Taylor et al. 2008). Con el objetivo de analizar los cambios producidos en la pesquería artesanal ocasionados por ENOS, se seleccionaron 5 especies de importancia comercial para la pesca artesanal en Ica entre 2004 y 2013, debido a los mayores desembarques registrados en el periodo de estudio, cuya relación con este evento natural no está claramente definida (Tabla 1). Estas tendencias ambientales pueden ser identificadas por el uso de Captura por Unidad de Esfuerzo (CPUE), como un índice de abundancia, y provee una manera importante para determinar decisiones de manejo efectivos (Fonteneau \& Richard 2003, Anticamara $e t$ al. 2011, Froese et al. 2012). Se emplearon modelos aditivos generalizados ajustados a CPUE de la pesquería artesanal, para examinar los impactos de ENOS sobre 1) la disponibilidad y 2) la abundancia de las 5 especies hasta dos años después de ocurrida sus anomalías, como una forma de contribuir hacia una gestión pesquera sostenible.

\section{Materiales y MÉTODOS}

Los datos de los desembarques diarios de la pesquería artesanal en el departamento de Ica, Perú (Fig. 1) entre 2004 y 2013 fueron proporcionados por el Instituto del Mar del Perú (IMARPE). Los datos por cada viaje fueron registrados siguiendo la metodología implementada por el proyecto 'Determinación del Potencial Pesquero Artesanal en el Litoral Peruano' e incluyen desembarque por especie $(\mathrm{kg})$, fecha de desembarque, arte y área de pesca (Estrella et al. 1999). Se calcularon los valores de la CPUE como captura $(\mathrm{kg})$ por viaje para cada una de las especies seleccionadas, siguiendo estudios previos (Estrella \& Swartzman 2010). Asimismo se empleó el principal arte de pesca para disminuir los errores en la CPUE.

Los datos de las anomalías de ENOS fueron obtenidos del Instituto Geofísico del Perú, como Índice Costero El Niño (ICEN; <http://www.met.igp.gob.pe/variabclim/indices.html>). El ICEN es un índice compuesto de la media corrida de 3 meses de anomalías de la temperatura superficial del mar (TSM) en la región 'Niño $1+2$ ' $\left(90^{\circ}-80^{\circ} \mathrm{W}, 10^{\circ} \mathrm{S}-0^{\circ}\right)$ con el objetivo de monitorear la fuerza de ENOS por la costera Peruana. Los valores del ICEN fueron representados para visualizar los cambios temporales en el ENOS (Fig. 2).

Tabla 1. Especies de importancia en la pesca artesanal de Ica, Perú analizados en el presente estudio

I Predominant fish species in the artisanal fisheries of Ica, Perú analyzed in this study

\begin{tabular}{llcl}
\hline Nombre común & Nombre científico & $\begin{array}{c}\text { Desembarques } \\
\text { 2004-2013 (ton) }\end{array}$ & $\begin{array}{c}\text { Arte de pesca } \\
\text { principal }\end{array}$ \\
\hline Cabinza & Isacia conceptionis & 6277,5 & Cerco \\
Cabrilla & Paralabrax humeralis & 519,8 & Pinta \\
Lisa & Mugil cephalus & 1060,7 & Cortina \\
Lorna & Sciaena deliciosa & 3960,8 & Cerco \\
Mojarrilla & Stellifer minor & 374,1 & Cortina \\
\hline
\end{tabular}



Figura 2. Anomalías mensuales de ENOS según el Índice Costero El Niño (ICEN), 2004-2013

/ ENSO anomalies according to the monthly Coastal El Niño Index (ICEN), 2004-2013 
Modelos aditivos generalizados o GAMs (Hastie \& Tibshirani 1986) fueron usados para modelar el impacto de ENOS. Los GAMs son comúnmente utilizados para modelar la influencia de variables ambientales sobre CPUE (Venables \& Dichmont 2004, Mitchell et al. 2014). Los valores de CPUE fueron transformados empleando el logaritmo natural para asumir una distribución normal. En dichos modelos se usaron 'splines' con el componente de disminución de 'double penalty' y fueron ajustados usando 'Restricted Máximum Likelihood' (REML), para un mejor rendimiento (Marra \& Wood 2011). ICEN fue incluido como predictor de CPUE para ver el efecto inmediato de ENOS sobre la disponibilidad de los recursos pesqueros. Para verificar si hay un intervalo entre ENOS y cambios en CPUE, como un proxy de abundancia, se ha incluido valores del ICEN con un lapso de 6, 12, 18, y 24 meses como predictores de CPUE. Tiempo (mes) y latitud fueron también incluidos para estandarizar los modelos contra cambios espaciotemporales naturales en la abundancia de los recursos, siendo el código GAM completo:

$\log ($ CPUE +1$) \sim \mathrm{s}($ ICEN $)+\mathrm{s}($ ICEN lapso 6 meses $)+\mathrm{s}($ ICEN lapso 12 meses $)+\mathrm{s}$ (ICEN lapso 18 meses $)+\mathrm{s}$ (ICEN lapso 24 meses $)+\mathrm{s}($ mes $)+\mathrm{s}($ latitud $)$

donde s son los 'splines'. Todos los nudos o 'knots' de los predictores fueron restringidos a 6 para reducir la sobresuavización. Deviance Explained (como $R^{2}$ ), significancia (valor de $P$ ), y Akaike Information Criterion (AIC) fueron calculados para cada modelo. Se utilizó el paquete ' $\mathrm{mgcv}$ ' del software estadístico libre R (R Development Core Team, versión 3.1.1., 2014, USA), con significancia en $P<0,01$.

\section{Resultados}

\section{Modelos GAMs}

Todos los modelos fueron fuertemente significativos explicando 5,1 a 38,4\% de la desviación en los datos (Tabla 2). Para Isacia conceptionis, Paralabrax humeralis, Mugil cephalus, Sciaena deliciosa, y Stellifer minor, cada predictor fue significativo $(P<0,01)$. Los coeficientes de correlación de Pearson entre los predictores indicaron que no habría problemas con multicolinealidad ( $-0,32<$ Pearson's $r<0,32)$. Todos los modelos fueron analizados según Wood (2006) y se determinó que no tenían sobre/bajo-suavización. Todos los modelos se ajustaron a la normalidad según diagnósticos. Para asegurar que el modelo sea reproducible, los GAMs están promediados a 0 .
Tabla 2. Resultados de los modelos GAM s/ Results of GAM modeling

\begin{tabular}{lccc}
\hline Especie & $\begin{array}{c}\text { Deviance } \\
\text { Explained } \\
(\%)\end{array}$ & AIC & Significancia \\
\hline I. conceptionis & 38,4 & 57244,09 & $P<2 \mathrm{e}^{-16}$ \\
$P$. humeralis & 16,6 & 37693,26 & $P<2 \mathrm{e}^{-16}$ \\
M. cephalus & 5,11 & 34284,30 & $P<2 \mathrm{e}^{-16}$ \\
S. deliciosa & 6,59 & 30708,37 & $P<2 \mathrm{e}^{-16}$ \\
S. minor & 12,2 & 33486,61 & $P<2 \mathrm{e}^{-16}$ \\
\hline
\end{tabular}

\section{Eventos de ENOS}

El ICEN entre 2004 y 2013 muestra la ocurrencia de periodos cálidos (El Niño) y fríos (La Niña) de manera intercalada, predominando periodos fríos a partir del 2007 (Fig. 2). Durante el periodo del estudio no se presentaron eventos El Niño intensos como los años 1982-83 y 1997-98 (i.e., ICEN > 2), pero se presentaron 3 eventos de La Niña moderados (i.e., ICEN $<-1)$.

\section{DisponibILIDAD de RECURSOS SEgún ENOS}

Para identificar las tendencias en la disponibilidad de recursos pesqueros según cambios en ENOS se empleó el ICEN sin un lapso temporal como predictor de la CPUE (Fig. 3). La CPUE de I. conceptionis fue elevada durante episodios moderados de La Niña y ligeros de El Niño, siendo menor durante periodos ligeros. P. humeralis presentó una disminución en su CPUE durante periodos de La Niña. $M$. cephalus fue elevada cuando los valores de ICEN estaban entre 0 y 1 (e.g., El Niño débiles) y alrededor de -2. La CPUE de $S$. deliciosa se vió desfavorecida por La Niña. De igual forma la CPUE de $S$. minor fue baja ante la presencia de La Niña (ICEN < -1,5).

\section{IMPACTOS DE ENOS SOBRE ABUNDANCIA EN EL LARGO PLAZO}

La CPUE es comúnmente usada como un índice de abundancia, y por tanto para conocer los impactos de ENOS sobre la abundancia de algunos recursos pesqueros en el largo plazo, se analizó el efecto del ICEN con un lapso temporal de 6, 12, 18, y 24 meses sobre la CPUE. La CPUE de $I$. conceptionis fue mayor durante los primeros 24 meses después de periodos moderados de La Niña, subiendo a los 12 meses tras un El Niño débil. $P$. humeralis fue baja después de los eventos de La Niña hasta 24 meses, teniendo una mayor CPUE después de El Niño débil, indicando una disminución de abundancia después de eventos de La Niña. La CPUE de $M$. cephalus fue baja después de eventos de La Niña. S. deliciosa 
a) I. conceptionis
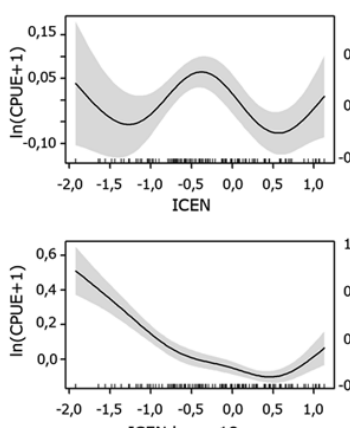

c) M. cephalus
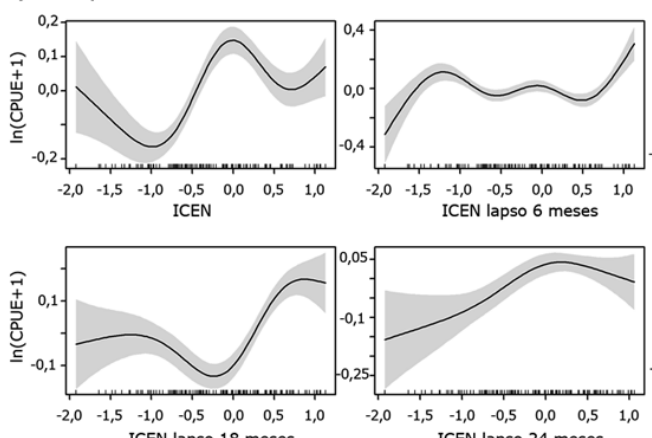

$-1,0-0,5$
ICEN lapso 6 meses

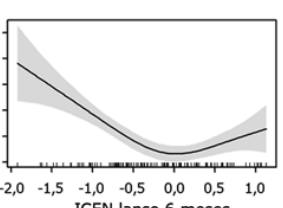

$\begin{array}{llll}-1,0 & -0,5 & 0,0 & 0,5 \\ \text { ICEN lapso } 6 \text { meses }\end{array}$

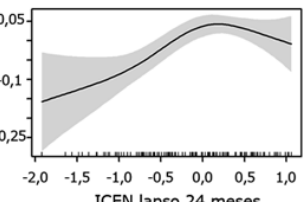

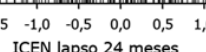

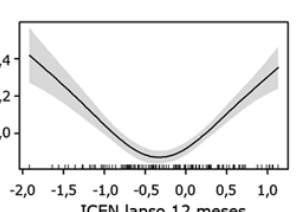

$\begin{array}{cccc}-1,0 & -0,5 & 0,0 & 0,5 \\ \text { ICEN lapso } 12 \text { meses }\end{array}$

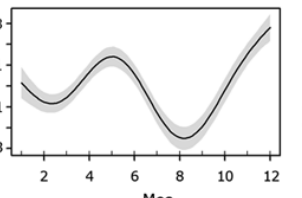

${ }^{6}$ Mes
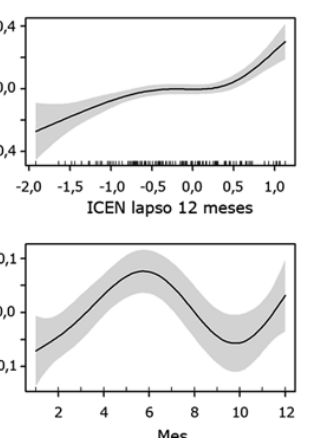

b) P. humeralis


ICEN lapso 18 meses
IC

d) S. deliciosa
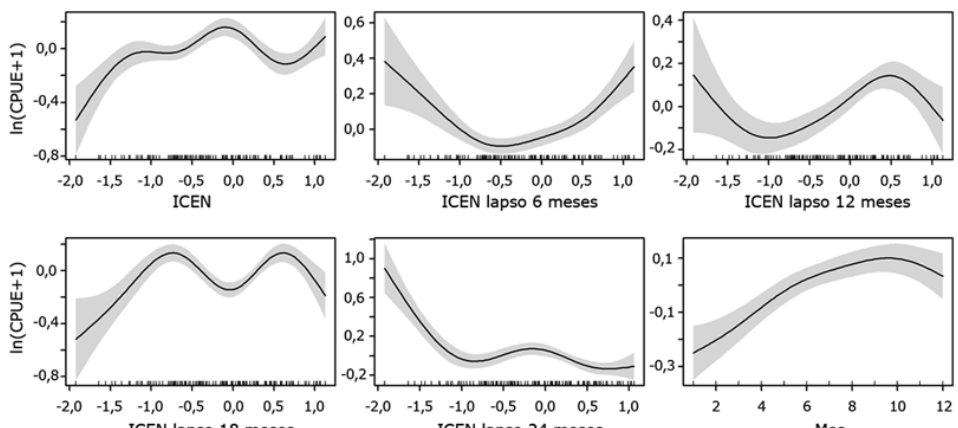
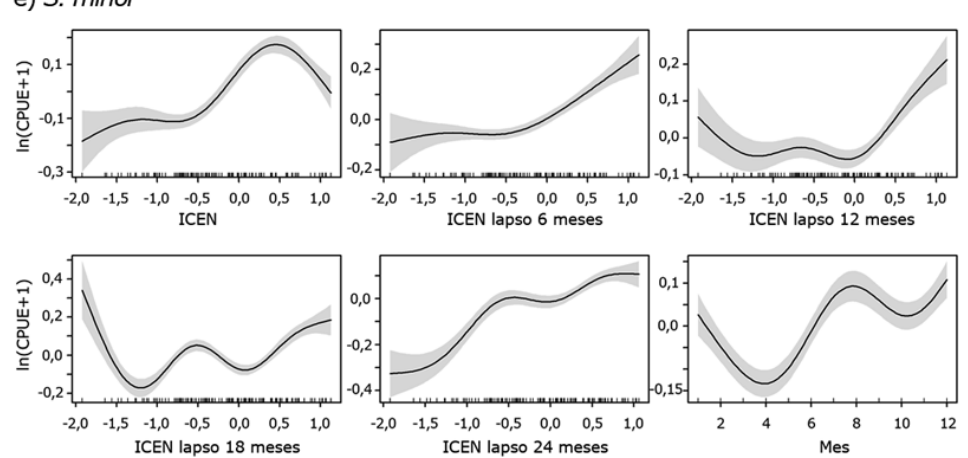

Figura3. are) Modelos GAM de CPUE (kgpor viaje) transformado logerítmicamente de los recursos pesquerosartesanalessegún predictores del Índice Costero 日 Niño (ICEN) con un lapso de resources as a function of the Coastal El Niño Index (ICEN) with a lag of 0, 6, 12, 18 and 24 months in Ica, Peru, 2004-2013 of I. conceptionis, P. humeralis, M. cephalus, S. deliciosa, and S. minor 
presentó CPUE variable respecto del ICEN con valores elevados a los 6, 12, y 24 meses tras eventos fríos de La Niña, pero a los 18 meses la CPUE fue baja respecto a los eventos de El Niño y La Niña. La respuesta de $S$. minor fue lo opuesto, con una CPUE baja tras eventos de La Niña con variación en el lapso de 18 meses.

\section{Discusión}

El análisis realizado muestra que ENOS afecta la disponibilidad inmediata de los recursos pesqueros artesanales mientras que la abundancia de estos es afectada en el largo plazo. En el periodo de estudio fueron observados varios ciclos de ENOS. Este periodo representa condiciones del régimen climático interdecadales de La Vieja, cuando la TSM está baja y eventos de La Niña -menos estudiada- son más comunes (Chávez et al. 2003). Dado el periodo de este estudio -que incluyen eventos La Niña y periodos normales-, la inclusión de modelos GAM y contar con una gran base de datos -tomada directamente de la actividad pesquera artesanal-, las tendencias de la pesquería de las especies estudiadas en relación a ENOS se muestran mejor. La inclusión de mes y latitud elimina la variación proveniente de patrones estacionales y espaciales de las especies. De la misma forma los datos analizados en este estudio incluyeron especies importantes para la población de pescadores artesanales que se encuentra en Ica, Perú; y, presentan por primera vez su relación cuantitativa con ENOS a largo plazo. Dada la variación en la 'Deviance Explained' entre los modelos, ENOS es más significativo respecto a $I$. conceptionis, $P$. humeralis y $S$. minor que $M$. cephalus y $S$. deliciosa.

Los modelos GAMs claramente demuestran que ENOS afecta profundamente la disponibilidad inmediata de recursos costeros de la pesca artesanal expresadas por la CPUE. En el caso de I. conceptionis y S. deliciosa, durante eventos El Niño ligeros y de La Niña fuertes, fueron asociados a una mayor CPUE. A diferencia de P. humeralis, M. cephalus, y S. minor donde los eventos de La Niña bajan la CPUE, similar a lo encontrado en estudios previos en el caso de $P$. humeralis (Espino 1990). De forma opuesta a Hoyos et al. (1983), quién encontró que $S$. minor disminuye durante episodios de El Niño en Ancón, tal vez por una migración horizontal entre las áreas del estudio. Dada la ausencia de eventos fuertes de El Niño durante nuestro periodo de estudio, no es posible conocer su impacto en S. minor. Durante eventos de La Niña, las aguas frías pueden ser aumentadas por el mayor afloramiento, ampliando la distribución de recursos y permitiendo menor acceso a los recursos pesqueros (Bertrand et al. 2004, Swartzman et al. 2008). Estos cambios en la distribución espacial de masas de agua también afectan la composición espacial del plancton (Ayón et al. 2008, Ochoa et al. 2010). Por ello durante El Niño o La Niña algunas especies que normalmente no están restringidas o asociadas a masas de agua o plancton, pueden encontrarse concentradas o con una distribución horizontal. Sin embargo, se requieren más estudios para identificar los mecanismos en las variaciones de CPUE de las especies incluidas respecto al ENOS.

Cambios en la abundancia de recursos pesqueros relacionado con ENOS son observados por respuestas en la CPUE de las especies a largo plazo. Este puede ser debido a variación en los procesos tales como el reclutamiento (Cahuin et al. 2009), dinámicas ecológicas (Wells et al. 2008), supervivencia de larvas y juveniles (Alheit \& Niquen 2004), reproducción (Mori et al. 2011, Claramunt et al. 2012), crecimiento, y mortalidad (Vilchis et al. 2005). En el caso de $I$. conceptionis, la abundancia (deducida por CPUE) fue mayor hasta 2 años después de ocurrido los eventos La Niña. De forma inversa, la abundancia de P. humeralis y $M$. cephalus se vió disminuida después de eventos La Niña y elevados luego de El Niño ligeros. La mayoría de las especies tienen tendencias temporales similares, marcando una respuesta biológica secuencial de la especie. Por ejemplo, la intensidad del impacto del ENOS sobre la CPUE de M. cephalus (visto por la escala del eje Y del modelo) baja con el tiempo. Por el contrario, la intensidad del impacto del ENOS sobre P. humeralis aumenta desde 6 meses hasta 2 años, probablemente debido al lapso entre ENOS y procesos que afectan el reclutamiento (Ñiquen $\&$ Bouchon 2004). En el caso de $S$. deliciosa y $S$. minor, las tendencias de CPUE después de 6, 12 y 24 meses de ENOS son similares, pero diferentes de las tendencias de CPUE después de 18 meses. Es poco probable que esta tendencia sea causada por fluctuaciones en la abundancia estacional, debido al empleo de la escala temporal (meses) en los modelos, y a que los eventos de ENOS no siguen fluctuaciones estacionales. Tal vez este patrón es el resultado de las diferencias de los impactos del ENOS entre cohortes.

Los cambios producidos por ENOS tienen una gran significancia para el manejo sostenible de los recursos pesqueros artesanales, especialmente considerando que los 'boom/auge' y 'bust/colapso' unidos a la variación climática son comunes en el Pacífico Sudoriental (Pauly \& Tsukayama 1987, ToralGranda 2008, Aranda 2009) y a la pesquería artesanal (Karnauskas et al. 2011, Thiao et al. 2012). Por ejemplo, después de un 'boom' de Argopecten purpuratus producido por un evento El Niño, la extracción aumentó enseguida por un 'bust' de la pesquería (Wolff et al. 2007). Estos resultados son un primer paso para informar y recomendar medidas de manejo que sean efectivas en especies donde el tamaño del stock desovante es un factor crucial en el reclutamiento de las 
siguientes generaciones. Sin embargo, se necesita profundizar los estudios sobre la biología y pesquería de estos recursos para entender mejor los impactos del ENOS y sus mecanismos subyacentes sobre la pesquería artesanal.

\section{Agradecimientos}

El presente estudio fue realizado con el apoyo de Héctor Sarmiento Bendezú, del Laboratorio Costero de Pisco - Instituto del Mar del Perú.

\section{LITERATURA CITADA}

Acha EM, HW Mianzan, RA Guerrero, M Favero \& J Bava. 2004. Marine fronts at the continental shelves of austral South America: Physical and ecological processes. Journal of Marine Systems 44: 83-105.

Alfaro-Shigueto J, JC Mangel, M Pajuelo, PH Dutton, JA Seminoff \& BJ Godley. 2010. Where small can have a large impact: Structure and characterization of small-scale fisheries in Peru. Fisheries Research 106: 8-17.

Alheit J \& M Niquen. 2004. Regimen shifts in the Humboldt Current ecosystem. Progress in Oceanography 60: 201-222.

Andrew N \& L Evans. 2009. Approaches and frameworks for management and research in small-scale fisheries in the developing world, $258 \mathrm{pp}$. The WorldFish Center Working Paper 1914. The WorldFish Center, Penang.

Anticamara JA, R Watson, A Gelchu \& D Pauly. 2011. Global fishing effort (1950-2010): Trends, gaps, and implications. Fisheries Research 107: 131-136.

Aranda M. 2009. Developments on fisheries management in Peru: The new individual vessel quota system for the anchoveta fishery. Fisheries Research 96: 308-312.

Ayón P, G Swartzman, A Bertrand, M Gutiérrez \& S Bertrand. 2008. Zooplankton and forage fish species off Peru: Large-scale bottom-up forcing and local-scale depletion. Progress in Oceanography 79: 208-214.

Bakun A \& SJ Weeks. 2008. The marine ecosystem off Peru: What are the secrets of its fishery productivity and what might its future hold? Progress in Oceanography 79: 290-299.

Ballón M, C Wosnitza-Mendo, R Guevara-Carrasco \& A Bertrand. 2008. The impact of overfishing and El Niño on the condition factor and reproductive success of Peruvian hake, Merluccius gayi peruanus. Progress in Oceanography 79:300-307.

Béné C, G Macfayden \& EH Allison. 2007. Increasing the contribution of small-scale fisheries to poverty alleviation and food security, $125 \mathrm{pp}$. Food and Agriculture Organization of the United Nations, Rome.

Bertrand, A, M Segura, M Gutiérrez \& L Vásquez. 2004. From small-scale habitat loopholes to decadal cycles: a habitat-based hypothesis explaining fluctuation in pelagic fish populations off Peru. Fish and Fisheries 5: 296-316.
Cahuin SM, LA Cubillos, M Ñiquen \& R Escribano. 2009. Climatic regimens and the recruitment rate of anchoveta, Engraulis ringens, off Peru. Estuarine, Coastal and Shelf Science 84: 591-597.

Cardinale M, O Chacate, M Casini, I Chaúca \& JH Vølstad. 2014. CPUE trends of Hilsa kelee and Thryssa vitrirostris exploited by the artisanal finfish fisheries in Mozambique derived from an on-shore sampling of catches by trip. Scientia Marina 78: 55-64.

Chavez FP, J Ryan, SE Lluch-Cota, CM Niquen. 2003. From anchovies to sardines and back: multidecadal change in the Pacific Ocean. Science 299: 217-221.

Claramunt G, LR Castro, LA Cubillos, H Hirche, G Perez \& M Braun. 2012. Inter-annual reproductive trait variation and spawning habitat preferences of Engraulis ringens off northern Chile. Revista de Biologia Marina y Oceanografia 47: 227-243.

Espino M. 1990. Análisis de las poblaciones de los principales recursos demersales del Perú. Boletín del Instituto del Mar del Perú 14: 3-26.

Espino M \& C Yamashiro. 2012. La variabilidad climática y las pesquerías en el Pacífico suroriental. Latin American Journal of Aquatic Research 40: 705-721.

Estrella C \& G Swartzman. 2010. The Peruvian artisanal fishery: changes in patterns and distribution over time. Fisheries Research 101: 133-145.

Estrella C, R Guevara-Carrasco, A Medina-Cruz, J Palacios-León, \& W Avila-Pérez. 1999. Informe estadístico de los recursos hidrobiológicos de la pesca artesanal por especies, artes, caletas y meses durante el segundo semestre de 1998. Informe del Instituto del Mar del Perú 143: 1-194.

Fonteneau A \& N Richard. 2003. Relationship between catch, effort, CPUE and local abundance for non-target species, such as billfishes, caught by Indian Ocean longline fisheries. Marine and Freshwater Research 54: 383-392.

Froese R, D Zeller, K Kleisner \& D Pauly. 2012. What catch data can tell us about the status of global fisheries. Marine Biology 159: 1283-1292.

Guisan A, TC Edwards \& T Hastie. 2002. Generalized linear and generalized additive models in studies of species distributions: setting the scene. Ecological Modeling 157: 89100.

Hastie T \& R Tibshirani. 1986. Generalized Additive Models. Statistical Science 1: 314-318.

Howell EA \& DR Kobayashi. 2006. El Niño effects in the Palmyra Atoll region: Oceanographic changes and bigeye tuna (Thunnus obesus) catch rate variability. Fisheries Oceanography 15: 477-489.

Hoyos L, J Tarazona, B Shiga \& V Chiong. 1983. Algunos cambios en la ictiofauna y sus relaciones tróficas durante el fenómeno el Niño en la Bahía de Ancón. Instituto del Mar del Perú, Boletín Volumen Extraordinario: 163-171 
INEI \& PRODUCE. 2012. I Censo de la pesca artesanal del ámbito marítimo 2012, 13 pp. INEI \& PRODUCE, Lima.

Karnauskas M, DB Mcclellan, JW Wiener, MW Miller \& EA Babcock. 2011. Inferring trends in a small-scale, datalimited tropical fishery based on fishery-independent data. Fisheries Research 111: 40-52.

Marra G \& SN Wood. 2011. Practical variable selection for generalized additive models. Computational Statistics and Data Analysis 55:2372-2387.

Mitchell JD, KJ Collins, PI Miller \& LA Suberg. 2014. Quantifying the impact of environmental variables upon catch per unit effort of the blue shark Prionace glauca in the western English Channel. Journal of Fish Biology 85: 657670.

Morales CE, SE Hormazábal \& J Blanco. 1999. Interannual variability in the mesoscale distribution of the depth of the upper boundary of the oxygen minimum layer off northern Chile (18-24S): Implications for the pelagic system and biogeochemical cycling. Journal of Marine Research 57: 909932.

Mori J, B Buitrón, A Perea, C Peña \& C Espinoza. 2011. Interannual variability of the reproductive strategy of the Peruvian anchovy off northern-central Peru. Ciencias Marinas 37(4B): 513-525.

Ñiquen M \& M Bouchon. 2004. Impact of El Niño events on pelagic fisheries in Peruvian waters. Deep-Sea Research Part II: Topical Studies in Oceanography 51(6-9): 563-574.

Ochoa N, MH Taylor, S Purca \& E Ramos. 2010. Intra- and interannual variability of nearshore phytoplankton biovolume and community changes in the northern Humboldt Current system. Journal of Plankton Research 32: 843-855.

Pauly D \& I Tsukayama. 1987. The Peruvian anchoveta and its upwelling ecosystem/: three decades of change, $351 \mathrm{pp}$. International Center for Living Aquatic Resources Management (ICLARM), Manila.

Quiñones J, VG Carman, J Zeballos, S Purca \& H Mianzan. 2010. Effects of El Niño-driven environmental variability on black turtle migration to Peruvian foraging grounds. Hydrobiologia 645: 69-79.

Salas S, R Chuenpagdee, JC Seijo \& A Charles. 2007. Challenges in the assessment and management of small-scale fisheries in Latin America and the Caribbean. Fisheries Research 87: 5-16.
Su NJ, CL Sun, AE Punt, SZ Yeh \& G Dinardo. 2011. Modelling the impacts of environmental variation on the distribution of blue marlin, Makaira nigricans, in the Pacific Ocean. ICES Journal of Marine Science 68: 1072-1080.

Swartzman G, A Bertrand, M Gutiérrez, S Bertrand \& L Vasquez. 2008. The relationship of anchovy and sardine to water masses in the Peruvian Humboldt Current System from 1983 to 2005. Progress in Oceanography 79: 228-237.

Taylor MH, M Wolff, J Mendo \& C Yamashiro. 2008. Changes in trophic flow structure of Independence Bay (Peru) over an ENSO cycle. Progress in Oceanography 79: 336351.

Thiao D, C Chaboud, A Samba, F Laloë \& P Cury. 2012. Economic dimension of the collapse of the 'False Cod' Epinephelus aeneus in a context of ineffective management of the small-scale fisheries in Senegal. African Journal of Marine Science 34: 305-311.

Toral-Granda V. 2008. Population status, fisheries and trade of sea cucumbers in Latin America and the Caribbean. In: ToralGranda V, A Lovatelli \& M Vasconcellos (eds). A global review of fisheries and trade. FAO Fisheries and Aquaculture Technical Paper 516: 213-229.

Venables WN \& CM Dichmont. 2004. GLMs, GAMs and GLMMs: an overview of theory for applications in fisheries research. Fisheries Research 70: 319-337.

Vilchis LI, MJ Tegner, JD Moore, CS Friedman, KL Riser, TT Robbins \& PK Dayton. 2005. Ocean warming effects on growth, reproduction, and survivorship of Southern California Abalone. Ecological Applications 15: 469-480.

Wells BK, JC Field, JA Thayer, CB Grime, SJ Bograd, WJ Sydeman, FB Schwing \& R Hewitt. 2008. Untangling the relationships among climate prey and top predators in an ocean ecosystem. Marine Ecology Progress Series 364: 1529.

Wolff M, M Taylor, J Mendo \& C Yamashiro. 2007. A catch forecast model for the Peruvian scallop (Argopecten purpuratus) based on estimators of spawning stock and settlement rate. Ecological Modelling 209: 333-341.

Wood SN. 2006. Generalized Additive Models: an introduction with R, 410 pp. Chapman and Hall/CRC, Boca Raton. 